\title{
The Efficacy of Adjuvant Intratympanic Steroid Treatment for Otitis Media with Effusion in Children
}

\author{
Hazem Saeed Amer ${ }^{1}$ Mohammad Waheed El-Anwar ${ }^{2} \quad$ Alaa Eldin Elfeky ${ }^{2}$ \\ ${ }^{1}$ Department of Otorhinolaryngology, Head and Neck Surgery, School \\ of Medicine, Zagazig University, Zagazig, Egypt \\ 2 Department of Otorhinolaryngology, Head and Neck Surgery, \\ Zagazig University, Zagazig, Egypt
Address for correspondence Hazem Saeed Amer, MD, Department of Otorhinolaryngology, Head and Neck Surgery, School of Medicine, Zagazig University, Zagazig 0020552322665 , Egypt \\ (e-mail: hazemamerent@yahoo.com).
}

Int Arch Otorhinolaryngol 2016;20:244-247.

\begin{abstract}
Introduction Otitis media with effusion (OME) is a leading cause of hearing impairment in children. Therefore, early and proper management is essential.

Objectives The objective of this research is to assess the efficacy of intratympanic (IT) steroids injection for management of otitis media with effusion (OME).

Methods This study involved 42 children (84 ears) with bilateral OME. We used tympanometry to confirm the childreńs middle ear effusion and pure tone audiometry to determine hearing threshold. We performed myringotomy and inserted ventilation tubes (VTs) bilaterally, followed by a steroid injection of $0.4-0.6 \mathrm{~mL}$ methylprednisolone $(40 \mathrm{mg} / \mathrm{mL})$ into one randomly selected middle ear. This procedure was followed by once-weekly administration of steroids $(0.5 \mathrm{~mL}$ methylprednisolone at a concentration of $40 \mathrm{mg} / \mathrm{mL}$ ) into the middle ear for three consecutive weeks.

Results We found recurrent OME after VT alone in nine (21.4\%) ears; whereas, after VT combined with steroid administration, we found two (4.76\%), with statistically significant difference. We noted tympanosclerosis postoperatively in six $(12.9 \%)$ ears and in one of the injected ears $(2.3 \%)(p=0.0484)$. Otorrhea occurred in eight $(19 \%)$ ears with VT alone and in three $(7.1 \%)$ injected ears, with non-significant difference. The duration between VT insertion and its extrusion was $6.6=1.1$ months for ears with VT alone and

Keywords

- middle ear effusion

- steroid

- tympanometry

$6.95=1.12$ months in injected ears ( $p=0.1541 \mathrm{NS})$.

Conclusion IT Steroids injection for treatment of OME is a safe and simple intervention with lower incidence of symptoms recurrence and postoperative complications. Thus, its use in management of OME is recommended.
\end{abstract}

\section{Introduction}

Otitis media with effusion (OME) is a leading cause of hearing impairment in children. Early and proper OME management prevents hearing and speech impairment in children because OME may be associated to developmental delay. However, OME treatment remains a controversial issue., ${ }^{1,2}$

received

April 22, 2015

accepted

August 5, 2015

published online

September 29, 2015
DOI http://dx.doi.org/

10.1055/s-0035-1564722. ISSN $1809-9777$.
Unfortunately, simple myringotomy and aspiration of effusion do not provide meaningful therapeutic results since their healing duration only lasts a couple of days. ${ }^{3,4}$ On the other hand, ventilation tube (VT) insertion may cause adverse effects, such as tube otorrhea, with a reported incidence rate ranging from 29 to $64 \%{ }^{5-7}$ Thus, conventional approaches

Copyright $(2016$ by Thieme Publicações License terms Ltda, Rio de Janeiro, Brazil
(1) $\Theta \circledast$ 
often fail to provide satisfactory and permanent relief of otologic symptoms. ${ }^{1,2,5,7}$

Systemic steroids are known to improve hearing levels in sudden sensorineural hearing loss (SSNHL), Menière's disease, and many other inner ear diseases. Intratympanic (IT) steroid injection has the potential to achieve higher steroid concentrations in the inner ear while avoiding the systemic side effects. 8,9

The aim of this study was to assess the efficacy of IT steroids for management of OME.

\section{Patient and Methods}

This study was performed between February 2013 and August 2014 and included 42 patients aged 3 to 11 years, with persistent bilateral OME and scheduled for surgery. The patients included necessarily had complaints of hearing loss and persistent bilateral OME after medical treatment lasting at least three months.

The patientś relatives provided written informed consent to share the results from this study, approved by the ethics committee. For recurrent cases after previous management by VT (grommet) insertion, the patients received radiotherapy. We excluded patients with mucocilliary or chronic medical disorders (such as diabetes mellitus).

We relied on tympanometry to confirm OME and on pure tone audiometry to determine hearing threshold. The children underwent this procedure over one to four days, preoperatively.

The patients underwent all surgeries under general anesthesia. The surgeon performed myringotomy using a sickle knife at the anteroinferior quadrant of the tympanic membrane (TM). Next, we aspirated the middle ear fluid.

A single surgeon performed the myringotomy and VT insertion bilaterally on each patient. Then, the surgeon prepared steroids for administration into one blindly selected middle ear. When necessary, the patient underwent adenotonsilectomy or adenoidectomy.

The patients were placed in a supine position with their heads turned $\sim 30$ degrees away from the surgeon. Using a syringe connected with a $22(3.5 \mathrm{IN} ; 0.7 \times 90 \mathrm{~mm})$ or 25 gauge spinal needle, the surgeon injected $0.4-0.6 \mathrm{ml}$ methylprednisolone (Depo-medrol ${ }^{\circledR} 40 \mathrm{mg} / \mathrm{mL}$, EPICO, Egypt and Pharmacia \& Upjohn, Belgium) into one tympanic cavity through the grommet tube under direct visualization through an operating microscope (-Fig. 1). The solution was not brought to body temperature before injection, given that the patient was under general anesthesia and, therefore, did not raise concerns of vertigo. The patient remained in the described position for $\sim 30$ minutes after injection to allow contact of steroid with the middle ear mucosa. Subsequently, patients received postoperative oral prophylactic antibiotic and were discharged from the hospital on the same day of surgery.

Follow-up examination happened once a week for three weeks and then monthly for six months. During the follow-up visit, steroids $(0.5 \mathrm{~mL}$ methylprednisolone; 10 drops at a concentration of $40 \mathrm{mg} / \mathrm{mL}$ ) in the form of ear drops was

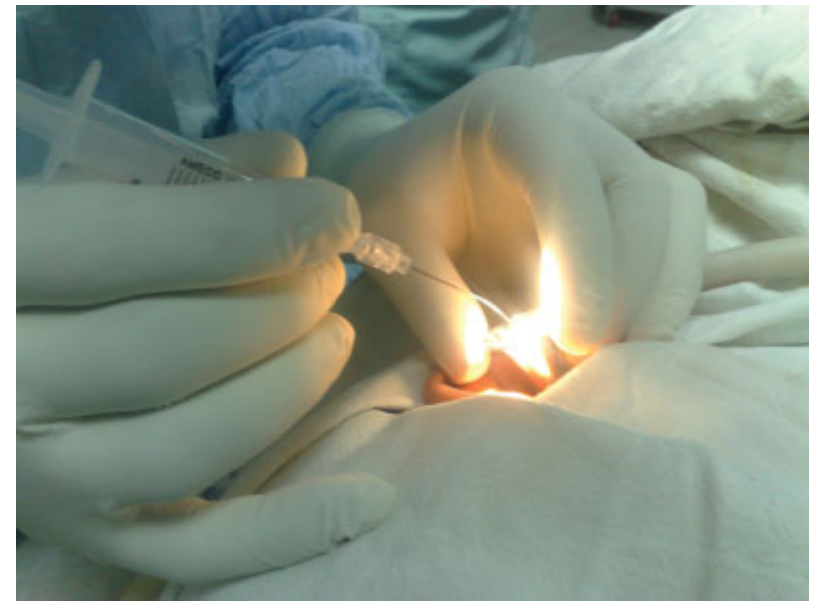

Fig. 1 Intratympanic steroid injection using a syringe connected to a spinal needle.

administered, through 5 drops into the previously injected ear once weekly for three consecutive weeks.

In this study, we define OME resolution (disease-free status) as the disappearance of aural fullness, normal findings on otoscopy, and type A tympanogram. ${ }^{2}$

We evaluated the recurrence of OME based on patient complaint of hearing loss, otoscopic criteria of OME, and audiotympanometry.

Diagnostic work in this study included Initial and 3 months later audiometry, tympanometry, and TM examination.

Patients were also evaluated for time of tube extrusion and complications such as tympanosclerosis, otorrhea, and persistent TM perforation.

We compared the results in both ears: the one with the grommet tube only and the one with the grommet tube and IT steroid.

Statistical comparisons and descriptive statistics were performed using the SPSS statistical software package (version 18.0; SPSS, Inc., Chicago, IL, USA). P value of $<0.05$ was considered statistically significant.

\section{Results}

Of the 42 patients ( 84 ears) included, there were 23 (54.8\%) males and $19(45.2 \%)$ females. Their age ranged from 3 to 11 years (-Table1). Hearing loss was the main complaint from all patients (100\%).

VT insertion was performed alone in $9(21.4 \%)$ patients and performed together with adenoidectomy in 21 (50\%); whereas adenotonsilectomy was needed in the remaining 12 (28.6\%) patients (-Table $\mathbf{1}$ ).

We detected different types of effusions operatively: mucoid effusion was found in 45 ears (53.6\%), while serous type was detected in 39 ears (46.4\%). With the exception of two, all patients had the same type of effusion in both ears with nonsignificant effect (chi square $=2.049 ; p=0.152$ ).

In the group treated with VT insertion only, mean hearing loss was $32.9 \pm 2.35 \mathrm{~dB}$ preoperatively and $13.9 \pm 2.7 \mathrm{~dB}$ postoperatively. As for the group treated with IT steroid 
Table 1 Surgical procedures, age and gender distribution

\begin{tabular}{|l|l|l|l|}
\hline \multicolumn{2}{|l|}{ Surgical procedure } & $\begin{array}{l}\text { Number } \\
\text { (42 patients, 84 ears) }\end{array}$ & Percent \\
\hline Ventilation tube (VT) alone & 9 & $21.4 \%$ \\
\hline VT+ adenoidectomy & 21 & $50 \%$ \\
\hline VT+ adenotonsilectomy & 12 & $28.6 \%$ \\
\hline \multirow{2}{*}{ Gender } & Male & 23 & $54.8 \%$ \\
\cline { 2 - 4 } & Female & 19 & $42.2 \%$ \\
\hline \multirow{2}{*}{ Age (years) } & Range & $3-11$ & \\
\cline { 2 - 4 } & Mean & n/a & \\
\hline
\end{tabular}

Table 2 Pre- and post-operative hearing threshold in ears with and without intratympanic steroid

\begin{tabular}{|l|l|l|l|l|}
\hline & Pre-treatment (dB HL) & Post-treatment (dB HL) & t-value & two-tailed $P$ value \\
\hline Without intratympanic steroid & $32.9 \pm 2.35$ & $13.9 \pm 2.7$ & 34.4 & $<0.0001 \mathrm{HS}$ \\
\hline With intratympanic steroid & $33.7 \pm 2.9$ & $10.6 \pm 3.1$ & 35.266 & $<0.0001 \mathrm{HS}$ \\
\hline t-value & 1.389 & 5.2023 & - & - \\
\hline two-tailed $p$-value & 0.1688 & $<0.0001 \mathrm{HS}$ & - & - \\
\hline
\end{tabular}

mean hearing loss was $33.7 \pm 2.9 \mathrm{~dB}$ preoperatively and $10.6 \pm 3.1 \mathrm{~dB}$ postoperatively. Thus, hearing improved significantly postoperatively in both groups $(p<0.0001)$. The mean postoperative hearing level was significantly higher after IT steroid application compared to VT only $(p<0.0001)$, taking into consideration that preoperative mean of hearing level of both groups showed non-significant differences $(p=0.1688)(-$ Table 2).

Tympanosclerosis was noted postoperatively in $6(12.9 \%)$ non-injected ears and one injected ear $(2.3 \%)$ and the difference was statistically significant $(p=0.0484)$. Otorrhea occurred in $8(19 \%)$ non-injected ears and $3(7.1 \%)$ injected ears with non-significant difference $(p=0.1)$. All patients that presented with otorrhea underwent conservative treatment, involving repeated suction, culture-guided topical antibiotic drops, and oral antibiotic for 10 days.

The mean (standard deviation, SD) duration between VT insertion and its extrusion was $6.6( \pm 1.1)$ for non-injected ears and $6.95( \pm 1.12)$ months in injected ears. This difference was not statistically significant $(p=0.1541)$ ( - Table 3 ).

Total reported recurrent OME was 9 (21.4\%) ears with VT alone and $2(4.76 \%)$ ears with VT and steroid injection. This difference was significant $(p=0.02)$. Both these patients with recurrent OME in their injected ear also had recurrent OME in the non-injected ear, which means that unilateral reported recurrent OME was 7 (21.4\%) in non-injected ears and 0 in injected ears, with very significant difference $(p=0.0057)$. Persistent TM perforation was not reported in any of the studied ears.

\section{Discussion}

OME is very common, especially in children. It is defined as middle ear effusion without signs and symptoms of an acute infection. OME can occur as a primary disorder or as a sequel of acute otitis media. ${ }^{10}$

Table 3 Differences between VT insertion only and VT insertion with IT steroid administration

\begin{tabular}{|l|l|l|l|l|l|l|}
\hline & \multicolumn{2}{|l|}{ VT insertion only } & \multicolumn{2}{l|}{ VT + IT steroid } & \multirow{2}{*}{ T test } \\
\cline { 2 - 6 } & Number of ears & Percent & Number of ears & Percent & \\
\hline Tympanosclerosis & 6 & $12.9 \%$ & 1 & $2.3 \%$ & $\mathrm{X}=3.896$ & $0.0484 \mathrm{~S}$ \\
\hline Otorrhea & 8 & $19 \%$ & 3 & $7.1 \%$ & $\mathrm{X}=2.615$ & $0.1 \mathrm{NS}$ \\
\hline $\begin{array}{l}\text { Mean time of tube } \\
\text { extrusion }\end{array}$ & $\begin{array}{l}6.95(1.13) \\
\text { months }\end{array}$ & $5-9.5$ months & $6.6(1.1)$ months & $4-9$ months & $\mathrm{t}=1.4383$ & $0.1541 \mathrm{NS}$ \\
\hline Recurrence OME & 9 & $21.4 \%$ & 2 & $4.76 \%$ & $\mathrm{X}=5.126$ & $0.02 \mathrm{~S}$ \\
\hline Unilateral recurrence & 7 & $16.7 \%$ & 0 & $0 \%$ & $\mathrm{t}=7.636$ & $0.0057 \mathrm{VS}$ \\
\hline
\end{tabular}

Abbreviations: IT, intratympanic; OME, otitis media with effusion; VT, ventilation tube. 
OME is a multifunctional disease. It has long been recognized that Eustachian tube (ET) dysfunction or obstruction predisposes to OME. Adenoids play an important role in its etiology. Cleft palate, Down's syndrome, Kartagner's syndrome, and OME associated with nasopharyngeal neoplasm, all direct to the role of ET dysfunction in OME. Immunologic and infectious factors are also included in its pathogenesis. ${ }^{11,12}$

Myringotomy and fluid aspiration alone are ineffective due to the short duration of the perforation healing time (usually only 1 to 2 days). ${ }^{3,4}$ On the other hand, the ventilation tube has an indwelling period of 4-6 months ${ }^{4-6}$ and is usually associated with a high incidence of postoperative middle ear infection and otorrhea, which could be refractory to local or medical treatment. ${ }^{6,7}$ Thus, the effectiveness of conventional methods for OME treatment remains questionable, presenting high recurrence rates that point to an unsolved issue.

Intratympanic delivery of medication has been making progress as a treatment method for various otologic disorders. In 1935, Barany used IT lidocaine for tinnitus. Schuknecht was among the first to use IT streptomycin for Meniere's disease. More recently, IT steroid delivery has been investigated in treating SSNHL. While IT steroid therapy has little systemic absorption, it provides a high local concentration of steroid. ${ }^{13}$

In our current study, we assessed the efficacy of IT steroids for management of OME in children (the main target in OME). It is nearly impossible to tolerate or accept local anesthesia or maintain IT position and most cases require adenoidectomy with or without tonsillectomy. Therefore, we used general anesthesia in this study, which allowed us to keep the patient in the needed position after IT steroid injection.

Furthermore, the use of VT in this study allowed drops to be applied weekly, since IT administration without need for transtympanic injection in a child is not possible without general anesthesia.

Adenoidectomy was needed in most (33) cases (78.6\%), as previously concluded. ${ }^{1}$ Nonetheless, we found that the most common predisposing factor for recurrence of OME was enlarged adenoid. ${ }^{1,5,6}$ This factor was neglected in this study, as we compare results between both sides of the same patient.

By reviewing the results of this study, it was clear that hearing improved significantly postoperatively in both groups. The mean postoperative hearing level was significantly higher after IT steroid application compared to VT, taking into consideration that preoperative mean of hearing level of both groups showed non-significant differences.

The results also clearly identified that IT steroid administration following VT insertion had significantly reduced the incidence of OME recurrence compared to VT insertion alone.

In this study, tympanosclerosis was significantly less in injected ears (2.3\%). This supports the autoimmune theory of OME and also explains this significant reduction of tympanosclerosis with steroid injection, despite the short follow-up period in this study. Although incidence of post-VT otorrhea in the current study was less with IT steroid use, this was statistically non-significant, indicating that otorrhea related only to patient and VT factors. Thus, IT steroid did not increase infection rates as occurred with systemic steroid.
Among the studied ears, the mean VT extrusion time in injected was 10 days longer than non-injected ears, with no significant difference. This reflects that IT steroid does not have an important role in increased tube duration.

Further studies are needed to evaluate the histopathological changes of middle ear mucosa after IT steroid injection in children with OME. More studies are also needed to find a method to reduce duration of the position maintained after IT injection, which prolongs general anesthesia.

\section{Conclusion}

Intratympanic steroids injection for treatment of OME is a safe and simple intervention that leads to less recurrence and postoperative complications. For this reason, the authors recommend its use for the management of OME.

\section{Conflict of Interest and Financial Disclosure}

The authors declare no financial support for this study and declare no conflict of interest.

\section{References}

1 Yousaf M, Inayatullah, Khan F. Medical versus surgical management of otitis media with effusion in children. J Ayub Med Coll Abbottabad 2012;24(1):83-85

2 Kuo CL, Wang MC, Chu CH, Shiao AS. New therapeutic strategy for treating otitis media with effusion in postirradiated nasopharyngeal carcinoma patients. J Chin Med Assoc 2012;75(7):329-334

3 Prokopakis EP, Lachanas VA, Christodoulou PN, Bizakis JG, Karatzanis AD, Velegrakis GA. Implications of laser assisted tympanostomy in adults. Otol Neurotol 2005;26(3):361-363

4 Sedlmaier B, Jivanjee A, Gutzler R, Huscher D, Jovanovic S. Ventilation time of the middle ear in otitis media with effusion (OME) after $\mathrm{CO}_{2}$ laser myringotomy. Laryngoscope 2002;112(4):661-668

5 Ho WK, Wei WI, Yuen AP, Hui Y, Wong SH. Otorrhea after grommet insertion for middle ear effusion in patients with nasopharyngeal carcinoma. Am J Otolaryngol 1999;20(1):12-15

6 Hwang CF, Chien CY, Lin HC, Peng JP, Chang HW, Su CY. Laser myringotomy for otitis media with effusion in nasopharyngeal carcinoma patients. Otolaryngol Head Neck Surg 2005;132(6): 924-927

7 Tang NL, Choy AT, John DG, van Hasselt CA. The otological status of patients with nasopharyngeal carcinoma after megavoltage radiotherapy. J Laryngol Otol 1992;106(12):1055-1058

8 Naganawa S, Satake H, Iwano S, Fukatsu H, Sone M, Nakashima T. Imaging endolymphatic hydrops at 3 tesla using 3D-FLAIR with intratympanic Gd-DTPA administration. Magn Reson Med Sci 2008;7(2):85-91

9 Nakashima T, Naganawa S, Sugiura M, et al. Visualization of endolymphatic hydrops in patients with Meniere's disease. Laryngoscope 2007;117(3):415-420

10 Burton MJ, Rosenfeld RM. Grommets (ventilation tubes) for hearing loss associated with otitis media with effusion in children. Otolaryngol Head Neck Surg 2006;135(4):507-510

11 Bernstein JM, Lee J, Conboy K, Ellis E, Li P. Further observations on the role of IgE-mediated hypersensitivity in recurrent otitis media with effusion. Otolaryngol Head Neck Surg 1985;93(5):611-615

12 Tomonaga K, Kurono Y, Chaen T, Mogi G. Adenoids and otitis media with effusion: nasopharyngeal flora. Am J Otolaryngol 1989; 10(3):204-207

13 Lustig LR. The history of intratympanic drug therapy in otology. Otolaryngol Clin North Am 2004;37(5):1001-1017 